\title{
Tizanidine-induced acute severe cystitis in a female taking famotidine
}

This article was published in the following Dove Press journal:

Clinical Pharmacology: Advances and Applications

29 July 2015

Number of times this article has been viewed

\section{Resham Raj Poudel' \\ Nisha Kusum Kafle ${ }^{2}$ \\ 'Department of Medicine, Om Saibaba Memorial Hospital, Kathmandu, Nepal; ${ }^{2}$ Department of Public Health, Institute of Medicine, Kathmandu, Nepal}

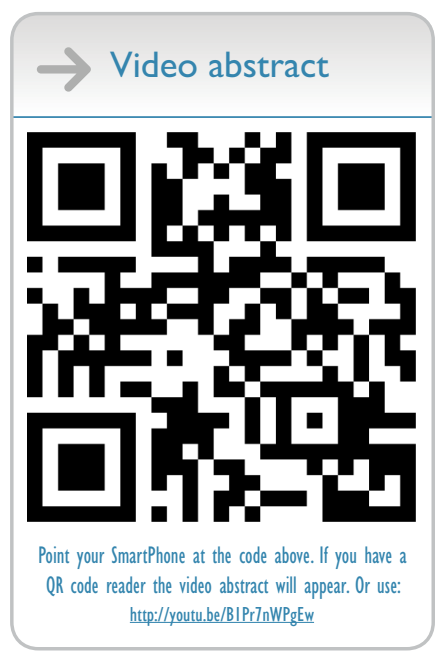

Correspondence: Resham Raj Poudel Om Saibaba Memorial Hospital, Kathmandu, Nepal

Tel +97798 6008 I539

Email poudelresham@gmail.com
Abstract: Cystitis is a possible adverse drug reaction associated with the use of tizanidine. Such cases have been rarely reported in literature because of the difficulty in establishing the causality. However, from a pharmacovigilance point of view, it is better to report such cases of a possible association between a drug's use and potential adverse drug reactions. We report a case of a 26-year-old Asian female on famotidine who presented with acute severe urinary burning after taking tizanidine.

Keywords: adverse drug reaction, cystitis, famotidine, tizanidine

\section{Introduction}

Tizanidine is a centrally acting $\alpha 2$ adrenergic agonist indicated for the management of spasticity. It is used as a muscle relaxant for spasticity caused by multiple sclerosis, amyotrophic lateral sclerosis (ALS), spinal injury, and stroke. ${ }^{1}$ As a muscle relaxant, tizanidine is as effective as baclofen and diazepam. ${ }^{2}$ The most common side effects of tizanidine treatment include drowsiness, hypotension, dry mouth, muscle weakness, and fatigue. ${ }^{3}$ Its prescription in patients taking antihypertensive medications needs careful consideration. ${ }^{4}$ It can also cause constipation and liver function test abnormalities. ${ }^{5}$ Cystitis is a less frequently occurring problem with tizanidine, which has been rarely reported. To our knowledge, the first case report of tizanidine-induced hemorrhagic cystitis was published in literature in $2013 .{ }^{6}$ We discuss here a case of a 26-year-old Asian female on famotidine $\left(\mathrm{H}_{2}\right.$ antihistaminic), who presented with acute severe dysuria after taking the second $2 \mathrm{mg}$ dose of tizanidine.

\section{Case presentation}

A 26-year-old Asian female presented to the emergency room for increased urinary frequency, severe pain, and burning overnight. She was unable to hold urine; however, she had no abdominal pain, blood in the urine, abnormal or cloudy urine color, strong or foul urine odor, or fever. Her menstrual periods had always been regular. She had a 5-year-old healthy child and no history of any obstetric or gynecological problems. She had a pulse rate of 90 beats/minute and blood pressure of 100/60 mmHg. Her body mass index was $22 \mathrm{~kg} / \mathrm{m}^{2}$. Investigations showed hemoglobin of $13.5 \mathrm{~g} / \mathrm{dL}$ (normal range: 12-16) and a total white blood cell count of $6,800 / \mathrm{mm}^{3}$ (normal range: 4,000-11,000); urine microscopy test did not reveal any red blood cells or white blood cells. Her kidney function tests were within normal limits. The woman had presented to an outpatient clinic the day before for neck pain and headache; with 
a diagnosis of torticollis, she was prescribed tizanidine $2 \mathrm{mg}$ three times a day and acetaminophen $500 \mathrm{mg}$ four times a day. She developed symptoms an hour after the second dose of tizanidine, ie, within 10 hours of initiation of the drug. She had been on famotidine $40 \mathrm{mg}$ once a day for 4 weeks for dyspepsia. She never had an episode of cystitis or urinary infections before. There was no history of any allergies or predisposing conditions that could have been responsible for the acute onset of this adverse event. Tizanidine treatment was subsequently discontinued and the patient was treated with hydration; her symptoms gradually improved within 24 hours. After stabilization, the patient was referred for urological consultation and ultrasonography of the urinary tract that failed to show any evidence of the current urinary problems. Urine culture did not yield any microorganism growth. The Naranjo probability scale was used to evaluate the possible causal relationship between tizanidine and the acute urinary symptoms. The scoring obtained by this algorithm was 4 , which indicates a possible causal association between the drug and the adverse reaction. The Naranjo probability scale categorizes adverse drug reaction (ADR) probability based on predefined weighted questions evaluating factors like temporal association of drug use and ADR occurrence, previous reports of ADR, alternative explanation of the event, drug level, dose-response relationship, etc. Scoring for Naranjo algorithm follows: $>9=$ definite ADR; $5-8=$ probable ADR; $1-4=$ possible ADR; $0=$ doubtful ADR. ${ }^{7}$

\section{Discussion}

Acute severe urinary symptoms can be alarming to both the patient and the health care provider, especially in the context of no clearly differentiating etiology. Non-infectious cystitis is most common in women of childbearing years, which may be idiopathic; or associated with bubble baths, feminine hygiene sprays, spermicidal jellies, radiation therapy to the pelvis; or consumption of certain foods, such as tomatoes, artificial sweeteners, caffeine, chocolate, and alcohol can also cause bladder symptoms. ${ }^{8}$ Tizanidine-induced cystitis is underreported due to challenges in establishing the causality with certainty; however, according to a key principle of pharmacovigilance, it is better to report even a suspicious case to generate an alarm in the interest of protecting public health. ${ }^{6}$ In multiple doses, in placebo-controlled studies using tizanidine, the incidence of urinary tract infection was $10 \%$ (7\% in placebo group) and urinary frequency was 3\% (2\% in placebo group). ${ }^{9}$ Between 2003 and 2012, the US Food and Drug Administration (FDA) reported 54 cases of hemorrhagic cystitis related to tizanidine use in the USA. ${ }^{6}$ Why tizanidine causes urinary burning and cystitis is not known. In our case, there was no predisposing factor for the urinary symptoms. However, if we assume these symptoms were a result of tizanidine use, there was concomitant use of famotidine, which could have increased the blood levels of tizanidine by inhibiting the CYP1A2 system, thereby decreasing its metabolism which could accentuate any effects attributable to tizanidine. Tizanidine is metabolized in the liver by CYP1A2, so drug interaction may occur when used with CYP1A2 inhibitors. Concomitant use of tizanidine with potent inhibitors of CYP1A2, fluvoxamine or ciprofloxacin, is contraindicated because of potential serious adverse effects. ${ }^{9-11}$ Less potent inhibitors of CYP1A2, eg, zileuton, other fluoroquinolones, antiarrythmics, cimetidine, famotidine, oral contraceptives, acyclovir, and ticlopidine should be used with caution. ${ }^{9}$ Given the very few case reports and the absence of references in the literature, this unique adverse effect of tizanidine needs a better consideration. Physicians, pharmacists, and other health care providers should be aware of this possible adverse event during treatment with tizanidine.

\section{Conclusion}

Tizanidine is a centrally acting $\alpha 2$ adrenergic agonist prescribed as a muscle relaxant in various causes of spasticity and pain. While dry mouth, dizziness, hypotension, and fatigue are frequently occurring side effects of the use of tizanidine, it has sometimes been associated with unexplainable symptoms of urinary infection and cystitis. Although rarely reported, health care providers and pharmacists should be aware of such possible reactions for better patient care. When etiology and mechanisms are uncertain, there is little opportunity for intervention; however, we can always help to minimize any drug reactions by closely following general precautions like avoiding drugs with contraindications, eg, the use of tizanidine with CYP1A2 inhibitors.

\section{Disclosure}

The authors have no conflicts of interest to disclose in this work.

\section{References}

1. Kamen L, Henney HR 3rd, Runyan JD. A practical overview of tizanidine use for spasticity secondary to multiple sclerosis, stroke, and spinal cord injury. Curr Med Res Opin. 2008;24(2):425-439.

2. Freitag FG. Preventative treatment for migraine and tension-type headaches: do drugs having effects on muscle spasm and tone have a role? CNS Drugs. 2003;17(6):373-381.

3. Simon O, Yelnik AP. Managing spasticity with drugs. Eur J Phys Rehabil Med. 2010;46(3):401-410. 
4. Johnson TR, Tobias JD. Hypotension following the initiation of tizanidine in a patient treated with an angiotensin converting enzyme inhibitor for chronic hypertension. J Child Neurol. 2000;15(12):818-819.

5. reference.medscape.com [homepage on the Internet]. Tizanidine (Rx)Zanaflex. Dosing Forms and Strengths. Medscape. Available from: http:// reference.medscape.com/drug/zanaflex-tizanidine-343071\#4. Assessed June 13, 2015

6. Palleria C, Leporini C, Chimirri S, et al. Limitations and obstacles of the spontaneous adverse drugs reactions reporting: Two "challenging" case reports. J Pharmacol Pharmacother. 2013;4(Suppl 1):S66-S72.

7. Naranjo CA, Busto U, Sellers EM, et al. A method for estimating the probability of adverse drug reactions. Clin Pharmacol Ther. 1981;30(2): 239-245.
8. US National Library of Medicine [homepage on the Internet]. Cystitis noninfectious. MedlinePlus. Available from: http://www.nlm.nih.gov/ medlineplus/ency/article/000514.htm. Accessed June 13, 2015.

9. Zanaflex ${ }^{\circledR}$ (tizanidine hydrochloride) capsules for oral use [prescribing information]. New York: Acorda Therapeutics Inc.; 2013.

10. Momo K, Homma M, Kohda Y, Ohkoshi N, Yoshizawa T, Tamaoka A. Drug interaction of tizanidine and ciprofloxacin: case report. Clin Pharmacol Ther. 2006;80(6):717-719.

11. Roberts RC, Part NJ, Pokorny R, Muir C, Leslie GC, Emre M. Pharmacokinetics and pharmacodynamics of tizanidine. Neurology. 1994;44(11 Suppl 9):S29-S31.

\section{Publish your work in this journal}

Clinical Pharmacology: Advances and Applications is an international, peer-reviewed, open access journal publishing original research, reports, reviews and commentaries on all areas of drug experience in humans. The manuscript management system is completely online and includes a very quick and fair peer-review system, which is all easy to use.
Visit http://www.dovepress.com/testimonials.php to read real quotes from published authors. 